\title{
Nonlinear surface electron transport over liquid helium
}

\author{
K.A. Nasyedkin, V.E. Sivokon, Yu.P. Monarkha, and S.S. Sokolov \\ B. Verkin Institute for Low Temperature Physics and Engineering of the National Academy of Sciences of Ukraine \\ 47 Lenin Ave., Kharkov 61103, Ukraine \\ E-mail: nasyedkin@ilt.kharkov.ua
}

Received May 14, 2009

\begin{abstract}
We present experimental data and a theoretical analysis of nonequilibrium mobility of surface electrons in liquid helium. The experiments are carried out in the temperature range where electron mobility is limited by electron scattering at surface excitations of liquid helium (ripplons). Holding and driving electric fields of wide ranges are used in measurements. Special attention is paid to the condition of strong holding fields under which hot electrons are confined to the ground surface level. Depending on the relation between the momentum relaxation rate and electron-electron collision frequency, different theoretical approaches are used to describe the nonlinear mobility of surface electrons. The results obtained allow to estimate the range of physical parameters where experimental data can be described by the theory of nonlinear electron transport within the ground surface level.
\end{abstract}

PACS: 68.03.-g Gas-liquid and vacuum-liquid interfaces;

73.20. $-\mathbf{r}$ Electron states at surfaces and interfaces;

73.25. $+\mathbf{i}$ Surface conductivity and carrier phenomena.

Keywords: liquid helium, surface electrons, two-ripplon process, electron conductivity.

\section{Introduction}

Surface electrons (SEs) on liquid helium represent a remarkable model system for studying transport phenomena in highly correlated two-dimensional (2D) electron liquids [1]. Since the energy relaxation rate of SEs induced by scattering at helium vapor atoms and quantized capillary waves (ripplons) is very low, the nonlinear transport conditions can be achieved already at weak driving electric fields $E_{\|}>1 \mathrm{mV} / \mathrm{cm}$. Usually, the areal density of SEs $n_{S}$ is changed by varying the holding electric field $E_{\perp}$ applied normally to the electron sheet. At the same time, it is very attractive to carry out the mobility measurements under conditions when the surface density $n_{s}$ and $E_{\perp}$ are independent parameters. This allows to study the SE transport for different relations between the momentum relaxation rate $v_{e-r}$ and the electron-electron collision frequency $v_{e-e}$. Moreover, keeping $n_{s}$ small under strong holding field conditions prevents evaporation of hot SEs to higher surface levels and allows to perform an accurate comparison with the theory of electron transport within the ground surface level.
In our previous work [2] the nonequilibrium SE mobility was measured at fixed temperature and surface density for different holding fields. The results were in a qualitative agreement with theory though there was a noticeable quantitative difference. That difference was a result of the lack of the measurement accuracy caused by difficulties in the experimental cell analysis. Later [3] an improvement of this analysis had given better numerical agreement with the theory at high holding fields.

In this work we present a systematic experimental study of electron mobility $\mu$ under nonequilibrium conditions induces by an ac driving electric field. The experiments are carried out at $T=0.52 \mathrm{~K}$ for $n_{s}=1.46 \cdot 10^{8} \mathrm{~cm}^{-2}$, $E_{\|}=1-20 \mathrm{mV} / \mathrm{cm}$, and $E_{\perp}=200-1400 \mathrm{~V} / \mathrm{cm}$. The experimental data of $\mu\left(E_{\|}\right)$are compared with theoretical results obtained using different approaches applicable for different ranges of the parameter $v_{e-e} / v_{e-r}$. Such a comparison allows to make important conclusions about mechanisms of momentum and energy relaxations of SEs and to reveal the influence of electron-electron collisions on nonlinear electron mobility resulting in specific regimes of electron transport. 
It is known that momentum and energy relations of hot SEs on liquid helium are governed by different scattering mechanisms. In particular, SE mobility is limited by oneripplon scattering processes involving long-wavelength ripplons with typical wave-vectors $q \lesssim 2 k \sim 10^{5} \mathrm{~cm}^{-1}$ (here $\mathbf{k}$ is the wave-vector of electrons). Such surface excitations have very low energy quanta $\hbar \omega_{q}<<T$ and, therefore, give very small contribution into SE energy relaxation. At the same time, energy relaxation is larger for two-ripplon scattering processes. In this case an electron can emit couples of short-wavelength ripplons with $2 \hbar \omega_{q} \sim T$ and a small total momentum $\left(\left|\mathbf{q}^{\prime}+\mathbf{q}\right| \lesssim 2 k\right)$. Such a difference between momentum and energy relaxations leads to interesting nonlinear dependencies of electron mobility on the driving electric field.

The article is organized as follows. In the Sec. 1, we describe the experimental setup and measurement procedure. Then, the theoretical approaches are described. The experimental results and comparison between experiment and theory are discussed. In Conclusion we summarize the results of this work.

\section{Experiment}

Mobility measurements were carried out using the experimental cell of circular geometry. The cell is described in detail in Ref. 2. The principal part of the cell is a plane capacitor situated horizontally. The charged surface of liquid helium is in the middle of the capacitor gap. The positive potential is applied to the bottom electrode to incite electrons to be trapped at the helium surface. The upper electrode is actually a set of measuring electrodes allowing to create an ac electric field of frequency $\omega$ in the electron layer and to measure an electron response to this field as an ac output voltage. The output voltage is a result of a current in electron layer, and therefore depends on complex conductivity of the layer. To find the relationship between the conductivity and the output-input signal ratio, we solved the Maxwell equations for electromagnetic field in the cell with appropriate boundary conditions [2]. As a result, two equations were found for two components of the cell conductance $G=G_{1}+i \omega G_{2}$. The conductance depends on real and imaginary components of electron layer inverse conductivity $\chi_{1}$ and $\chi_{2}$ :

$$
G_{1}=G_{1}\left(\chi_{1}, \chi_{2}, \omega, A\right) ; \quad G_{2}=G_{2}\left(\chi_{1}, \chi_{2}, \omega, A\right)+G_{0} .
$$

Here $A$ denotes parameters depending on the cell geometry, $G_{0}$ is the empty cell conductance. The solution of Maxwell equations allows also to estimate the radial distribution of the electric field operating in the electron layer and depending on the layer conductivity.

The cell conductance can be easily obtained from the output-input signal ratio, taking into account parameters of the measuring line found independently. Therefore, in order to find the inverse conductivity of the electron sheet i.e., $\chi_{1}$ and $\chi_{2}$ one needs to solve the system of Eqs. (1).

It should be noted that the real experimental cell is some different from the idealized one, described above using Maxwell equations. The difference appears, for example, when comparing the calculated and measured values of the empty cell conductance. Such a difference is possibly arisen due to intractable parasitic electric links between the electrodes and the grounded cell body. However the main difference between the real and idealized experimental cells is due to grounding the central measuring electrode. This grounding can be taken into account if one multiplies the values of $G_{1}, G_{2}$, and $G_{0}$ by the $S_{\text {out }} /\left(S_{\text {out }}+S_{\text {ground }}\right)$, where $S_{\text {out }}$ and $S_{\text {ground }}$ are the surfaces of the output and grounded electrodes, respectively. To account extra differences, we introduce a fitting impedance between the input and ground electrodes and use such an impedance in all our calculations.

When SEs are overheated by the driving electric field, electron mobility is high and, therefore, one have to measure rather small phase changes. To reduce the influence of uncontrolled factors, the empty cell conductance was measured under the same experimental conditions and in the same runs as those of the cell with electrons. The $G_{0}$ in Eqs. (1) reflects a connection between the input and output electrodes, which does not depend on the electron layer conductivity, and the relevant part of the cell conductance should be subtracted of the solution of Eqs. (1).

The presence of the electron layer in the cell changes, due to screening effects, the connection between the input and output electrodes. Also it changes the parasitic links between the electrodes and the cell body. Therefore to solve Eqs. (1) properly, a correction to the value of $G_{0}$ is necessary. The correction was made for every measurement basing on the criterion that the solution of Eqs. (1) should give a proper value of the imaginary part of the inverse conductivity $\chi_{2}$. Note that the value of $\chi_{2}$ is proportional to the effective electron mass which is equal to the free electron mass if electron temperature is higher than the Wigner solid melting temperature.

The stability of solutions of Eqs. (1) is limited by the measurement accuracy since $\chi_{2}<<\chi_{1}$. Because of this inequality the value of $\chi_{2}$ rather reflects the inaccuracy of measurements than the physically-meaning imaginary part of inverse conductivity. Previously, when solving Eqs. (1) $[2,3]$ either the value $\chi_{2}$ was disregarded or the set of Eqs. (1) was reduced to a single equation for certain chosen value of $\chi_{2}$. The method of calculation based on solving the set of Eqs. (1) used in the present work and that of solving the reduced single equation give almost the same results. Nevertheless we prefer the first method as more strict.

To simplify the analysis of results initial data were approximated by a smooth curve before being used for esti- 

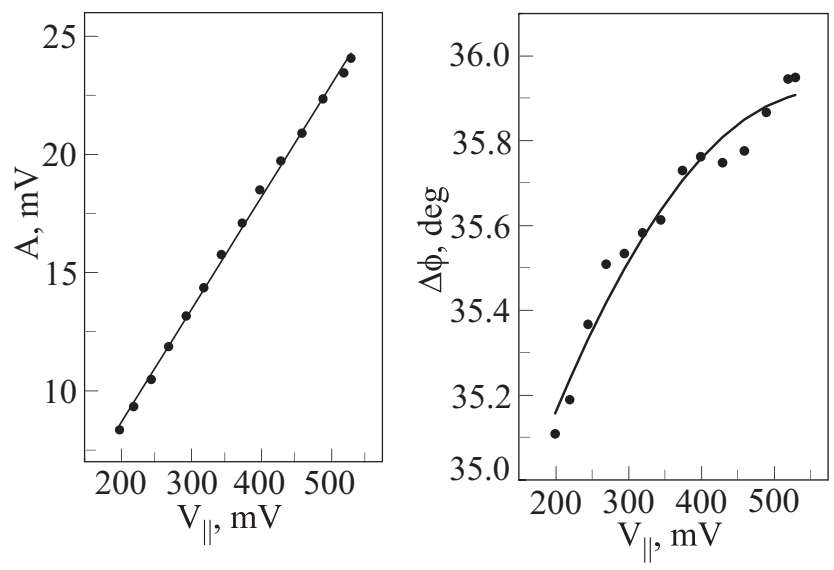

Fig. 1. The amplitude $A$ and phase shift $\Delta \phi$ of the empty cell as a function of driving potential.

mation of the cell conductance under solution of Eqs. (1). Results of averaging of four measurements series for the empty cell are presented in Fig. 1. The phase change and output voltage amplitude are measured for different amplitudes of the input voltage (circles). The measurements are performed in temperature range $T=0.55-0.76 \mathrm{~K}$. In addition to quite expected linear dependence between the input and output amplitudes the increase of phase with increasing the input voltage is observed. The possible reason for such nonphysical dependence may be a nonlinearity in the measuring circuits appearing as a result of difference between real and idealized cells. As we noted above, to take this dependence into account, the fitting impedance was used in data processing. The smoothing of the experimental data is demonstrated by lines in Fig. 1. Note that we did not observe the influence of the holding potential on the conductance $G_{0}$ of the empty cell.
The approximation of the initial data under evaluation of SE mobility was also performed. As an example, the data on electron mobility at the holding field $1190 \mathrm{~V} / \mathrm{cm}$ are shown in Fig. 2. Fig. 2, $a$ and 2,b illustrate, respectively, the relationship between the output voltage phase shift and amplitude and the input voltage amplitude. Points indicate the experimental data and lines are results of the approximation used in further calculations. The estimated electron mobility is shown in Fig. 2,c (solid line). The dashed and dotted lines correspond to theoretical calculation in one-electron and complete control approximations, respectively (we discuss these approaches in next Section). The points are experimental data obtained without the initial data approximation. They illustrate data errors. The errors increase with decreasing the holding potential and increasing the electron mobility increase.

Regarding evaluation of the driving field operating in the electron sheet plane we should like to note the following. The solution of Maxwell equations allows to calculate the radial distribution of the driving field in the electron layer $E_{\|}(r)$. It is essential that the field value depends on the layer conductivity. The solution of Eqs. (1) was obtained for the linear regime where the relation between local current and local field is given by Ohm's law and the conductivity does not depend on $E_{\|}$[2].

In present work, we study the nonlinear conductivity which depends on the field. Under such condition the solution of Eqs. (1) obtained in Ref. 2 does not correspond exactly to experimental situation. Though there are two factors which justify using the linear calculation in the nonlinear case. First, high rate of electron-electron relaxation prevents temperature gradients in the electron layer so it is quite possible that the field distribution differs unessentially from the linear mode at some averaged value
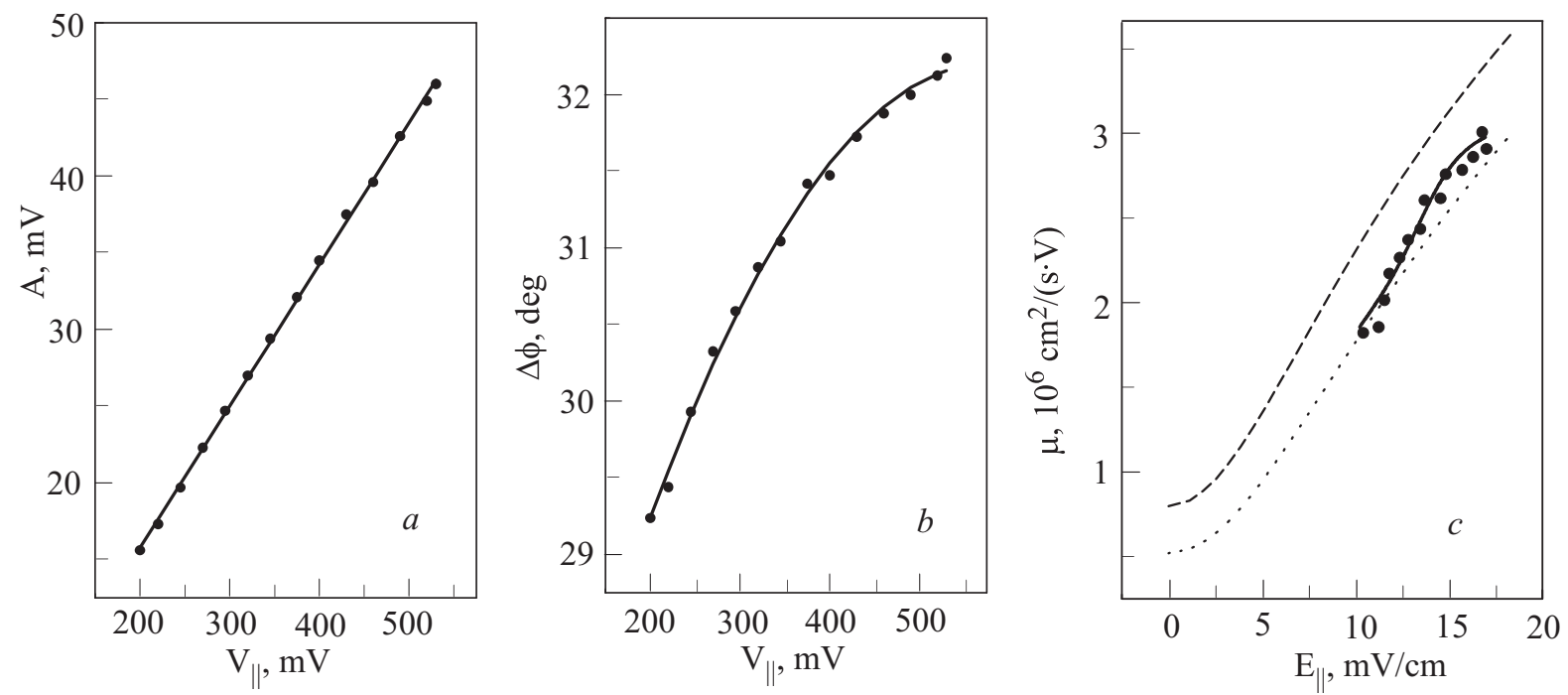

Fig. 2. The amplitude $A\left(V_{\|}\right)(a)$, phase shift $\Delta \phi\left(V_{\|}\right)(b)$ for the experimental cell with surface electrons, and SE mobility as a function of driving field $E_{\|}(c)$ for holding field $E_{\perp}=1190 \mathrm{~V} / \mathrm{cm}$. Points indicate the experimental data and lines are results of the approximation. The dashed and dotted lines correspond to theoretical calculations in one-electron and complete control approximations, respectively. 
of the driving field. Second, the driving field influence on the conductivity is not drastic even though being well-pronounced. Therefore the linear solution can be a quite reasonable approximation to the actual experimental conditions though the level of errors in this case is not clear a priori.

In our work, the mobility of electrons is measured as a function of driving electric field which is a result of some averaged radial driving electric field across the cell taking into account its dependence on layer conductivity. We estimated such an averaged field as

$$
\bar{E}_{\|}=\frac{1}{R_{\mathrm{el}}} \int_{0}^{R_{\mathrm{el}}}\left|E_{\|}(r)\right| d r .
$$

Here $R_{\mathrm{el}}$ is the radius of electron layer.

\section{Theory}

\subsection{Mobility as a function of the effective electron temperature}

We shall confine ourselves to electron scattering within the ground surface level only. In this case the wave function of a SE is usually written as $\psi_{1, \mathbf{k}}(z, \mathbf{r})=$ $=2 \gamma_{1}^{3 / 2} z \exp \left(-\gamma_{1} z+i \mathbf{k r}\right)$, where the parameter $\gamma_{1}$ is found by variation to minimize the ground energy. For zero holding field, $\gamma_{1} \rightarrow \gamma_{1}^{(0)}=m \Lambda_{0} / \hbar^{2}$, where $\Lambda_{0}=$ $=\left(e^{2} / 4\right)(\epsilon-1) /(\epsilon+1), \epsilon$ is the liquid helium dielectric constant, $e$ and $m$ are electron charge and mass, respectively. The above given wave-function is found assuming that the electron potential barrier at the interface $V_{0} \rightarrow \infty$. For the real value of $V_{0} \simeq 1 \mathrm{eV}$, penetration of the electron wave-function into liquid helium is described by the parameter $\kappa_{0} \simeq \sqrt{2 m V_{0}} / \hbar \simeq 5 \cdot 10^{7} \mathrm{~cm}^{-1}$ which is much larger than $\gamma_{1}$.

SEs on liquid helium represent a highly correlated electron liquid of which the average Coulomb interaction energy is much larger than the average kinetic energy. This means that an electron is frequently reflected by a repulsion potential barrier of other electrons. The frequency of such electron-electron collisions $v_{e-e}$ can be estimated as a frequency of harmonic oscillations in the field of other electrons fixed in a 2D triangular lattice $\omega_{0}=2.1 \sqrt{e^{2} / m} n_{s}^{3 / 4}$ [4]. This frequency is always much higher than the electron energy relaxation rate $\widetilde{v}_{e-r}$ which is about $10^{6} \mathrm{~s}^{-1}$ at $T_{e} \sim 1 \mathrm{~K}$. Therefore, in the nonlinear transport experiment SEs can be characterized by an effective electron temperature $T_{e}$ which can be substantially higher than the ambient temperature $T$.

Regarding momentum relaxation which is much higher than energy relaxation, two opposite extreme regimes can be realized for SEs on liquid helium: $v_{e-e}<<v_{e-r}$ and $v_{e-e} \gg v_{e-r}$. In the first regime, electron momentum relaxation is governed by electron-ripplon scattering, and the momentum distribution function is found as a solution of the kinetic equation for noninteracting SEs. The effective electron temperature is just a parameter found from the energy balance equation. In the second regime, momentum relaxation is governed (controlled) by electron-electron collisions, and the momentum distribution function is just the equilibrium distribution function $f_{0}\left[\left(\varepsilon_{k}-\hbar \mathbf{k u}\right) / T_{e}\right]$ shifted according to the drift velocity $\mathbf{u}$ (here $\varepsilon_{k}=$ $=\hbar^{2} k^{2} / 2 m$ ). The drift velocity is found from the total momentum balance equation. This regime is sometimes called as the complete control regime. Because of extremely low energy relaxation, the main nonlinear effect comes from electron heating $\left(T_{e}>T\right)$. The other nonlinear parameter $\hbar \mathbf{k u} / T_{e}$ is assumed to be small. In this case, we can expand the distribution function in $\hbar \mathbf{k u} / T_{e}$ only up to the linear term, keeping in mind that the effective collision frequency (momentum relaxation rate) now depends on the electron temperature $T_{e}\left(E_{\|}\right)$.

The above described two approaches lead to different mobility equations. The mobility found in the single-electron treatment is given by

$$
\mu^{(1 e)}=\frac{e}{m} \int_{0}^{\infty} \frac{x \exp (-x)}{v_{e-r}^{(1 e)}(x)} d x,
$$

where

$$
v_{e-r}^{(1 e)}(x)=v_{0} \frac{T_{e}}{T} \frac{J(x)}{x} ; v_{0}=\frac{8 \gamma_{0}^{2} T^{2}}{\pi \hbar \alpha} ; \gamma_{0}=\frac{m \Lambda_{0}}{\hbar^{2}} .
$$

The dimensionless function $J(x)$ is defined by electronripplon coupling

$$
\begin{gathered}
J(x)=x^{2} \int_{0}^{\pi / 2} \varphi^{2}\left(\sqrt{\frac{T_{e} x}{\Delta_{1}}} \sin \theta\right) \sin ^{4} \theta d \theta+ \\
+\frac{2}{3}\left(\frac{\Delta_{\perp}^{3}}{\Delta_{0} T_{e}^{2}}\right)^{1 / 2} x \int_{0}^{\pi / 2} \varphi\left(\sqrt{\frac{T_{e} x}{\Delta_{1}}} \sin \theta\right) \sin ^{2} \theta d \theta+\frac{\pi}{18} \frac{\Delta_{\perp}^{3}}{\Delta_{0} T_{e}^{2}},
\end{gathered}
$$

where

$$
\varphi(y)=-\frac{1}{1-y^{2}}+\frac{1}{\left(1-y^{2}\right)^{3 / 2}} \ln \left(\frac{1+\sqrt{1-y^{2}}}{y}\right),
$$

$\Delta_{0(1)}=\hbar^{2} \gamma_{0(1)}^{2} /(2 m)$ and we introduce the notation $\Delta_{\perp}=\hbar^{2} \gamma_{\perp}^{2} /(2 m)$ where $\gamma_{\perp}^{3}=3 m e E_{\perp} /\left(2 \hbar^{2}\right)$. The function $\varphi(y)$ describes the contribution from polarization interaction between SEs and interface displacements [5]. If one applies the approximation $\varphi(y) \simeq 1 /(3 y)$ numerically justified for $y \sim 1$, the expression for $J(x)$ simplifies considerably and $v_{e-r}^{(1 e)}(x)$ is now reads as 


$$
\begin{gathered}
v_{e-r}^{(l e)}(x) \simeq \frac{e^{2}}{4 \hbar \alpha}\left[E_{p}^{2}+\frac{4 \sqrt{2}}{\pi}\left(\frac{T}{T_{e}}\right)^{1 / 2} \frac{E_{p} E_{\perp}}{\sqrt{x}}+\left(\frac{T}{T_{e}}\right) \frac{E_{\perp}^{2}}{x}\right] \\
E_{p}=\frac{2 \Lambda_{0} \gamma_{1} \sqrt{m T}}{3 \hbar e}
\end{gathered}
$$

At $T \simeq 0.5 \mathrm{~K}$ the typical value of $E_{p}$ is about $300 \mathrm{~V} / \mathrm{cm}$.

For the complete control regime $\left(v_{e-e}>>v_{e-r}\right)$ the many-electron expression for SE mobility can be found as

$$
\mu^{(m e)}=\frac{e}{m v_{e-r}^{(m e)}},
$$

where the effective collision frequency $v_{e-r}^{(m e)}$ is defined by

$$
\begin{gathered}
v_{e-r}^{(m e)}=\frac{\sqrt{\pi}}{2} v_{0}\left(\frac{T_{e}}{T}\right)\left\{\int_{0}^{\infty} x^{3 / 2} \varphi^{2}\left(\sqrt{\frac{T_{e} x}{\Delta_{1}}}\right) \exp (-x) d x+\right. \\
\left.+\frac{2}{3}\left(\frac{\Delta_{\perp}^{3}}{\Delta_{0} T_{e}^{2}}\right)^{1 / 2} \int_{0}^{\pi / 2} x^{1 / 2} \varphi\left(\sqrt{\frac{T_{e} x}{\Delta_{1}}}\right) \exp (-x) d x+\frac{\sqrt{\pi}}{9} \frac{\Delta_{\perp}^{3}}{\Delta_{0} T_{e}^{2}}\right\} .
\end{gathered}
$$

For $\varphi(y) \simeq 1 /(3 y)$, the Eq. (7) is reduced to

$$
v_{e-r}^{(m e)}=\frac{e^{2}}{4 \hbar \alpha}\left[E_{p}^{2}+\frac{4}{\sqrt{2 \pi}}\left(\frac{T}{T_{e}}\right)^{1 / 2} E_{p} E_{\perp}+\left(\frac{T}{T_{e}}\right) E_{\perp}^{2}\right]
$$

Consider asymptotic limits of SE mobility as a function of $E_{\perp}$ given by Eqs. (2)-(8). In the limit $E_{\perp}<<E_{p}$, where electron-ripplon scattering is due to polarization interaction between SEs and interface displacements, one obtains

$$
\mu^{(l e)} \simeq \mu^{(m e)} \simeq \frac{9 e \alpha \hbar^{3}}{m^{2} \Lambda_{0}^{2} \gamma_{0}^{2} T} .
$$

The asymptote (9) describing SE mobility within the ground level $l=1$ does not depend on $T_{e}$ and hence on the driving electric field even for hot electrons. This property was firstly found using one-electron approach [6]. For the equilibrium case $\left(T_{e}=T\right)$, the relationship between $\mu^{(1 e)}$ and $\mu^{(m e)}$ given by Eq. (9) was obtained and observed experimentally for $E_{\perp}<<E_{p}$ and different electron densities $[7,8]$. Of course, heating of SEs by the driving electric field can make $T_{e}$ comparable with the energy difference between the ground and excited surface levels. This leads to evaporation of SEs from the ground surface level and to a sharp increase of their mobility $[9,10]$ even at low driving fields. Under such conditions, instead of Eq. (6), we have a more complicated dependence of electron mo- bility on $T_{e}$, which was found qualitatively using certain approximations and simplifications.

In the opposite limit $E_{\perp}>E_{p}$, the contribution from the holding field term of the electron-ripplon coupling predominates, and SE mobility is given by

$$
\mu^{(1 e)} \simeq 2 \mu^{(m e)} \simeq \frac{8 \hbar \alpha T_{e}}{m e E_{\perp}^{2} T}
$$

In this case, SE mobility within the ground surface level is a linear function of $T_{e}$ in agreement with Ref. 11, and $\mu^{(m e)}$ found for $v_{e-e}>>v_{e-r}$ is half as much the single-electron mobility $\mu^{(l e)}$. The experimental confirmation of mobility expressions of Eqs. (9) and (10) under equilibrium conditions $\left(T_{e}=T\right)$ for different holding fields had given the evidence of realization of the complete control regime in the system of SEs on liquid helium $[7,8]$.

Giving correct theoretical description of SE mobility in the extreme regimes $\left(v_{e-e}<<v_{e-r}\right.$ and $\left.v_{e-e}>>v_{e-r}\right)$ the results of Eqs. (2)-(10) are not suitable in the intermediate case when $v_{e-e} \sim v_{e-r}$. One can expect a smooth transition between these two asymptotes when varying the parameter $v_{e-e} / v_{e-r}$ by changing electron density or the holding electric field. A theoretical description of SE mobility applicable for arbitrary relation between $v_{e-e}$ and $v_{e-r}$ was found in Ref. 12:

$$
\mu=\mu^{(m e)}\left(\frac{v_{e-r}^{(m e)}+v_{e-e}}{\bar{v}_{e-r}^{(1 e)}+v_{e-e}}\right),
$$

where $\bar{v}_{e-r}^{(1 e)}=e / m \mu^{(1 e)}$. This expression reproduces Eqs. (6) and (2) as the opposite limiting cases $v_{e-e}>>v_{e-r}^{(m e)}$, $\bar{v}_{e-r}^{(1 e)}$ and $v_{e-e}<<v_{e-r}^{(m e)}, \bar{v}_{e-r}^{(1 e)}$, respectively. Fitting experimental data by Eq. (11) one can estimate $v_{e-e}$ for different holding and driving electric fields.

\subsection{Energy relaxation}

In our experiment, the SE mobility $\mu\left(E_{\|}\right)$was measured as a function of the driving electric field. In order to compare data with theoretical evaluations of $\mu\left(T_{e}\right)$, we use the energy balance equation which establishes the relationship between $E_{\|}$and $T_{e}$ :

$$
e \mu\left(T_{e}\right) E_{\|}^{2}=\dot{Q},
$$

where $\dot{Q}$ is the energy transferred by an electron to ripplons per unit time. For electron scattering which involves two ripplons with $\left|\mathbf{q}^{\prime}+\mathbf{q}\right|<<q, q^{\prime}$, it is given by [14]

$$
\begin{gathered}
\dot{Q} \simeq \frac{m}{2 \pi \rho^{2}} \int_{0}^{\infty} W^{2}(q) \frac{q^{3}}{\omega_{\mathbf{q}}}\left(N_{\mathbf{q}}+1\right)^{2} \times \\
\times\left[\exp \left(-\frac{2 \hbar \omega_{\mathbf{q}}}{T_{e}}\right)-\exp \left(-\frac{2 \hbar \omega_{\mathbf{q}}}{T}\right)\right] d q,
\end{gathered}
$$


where $W(q)$ is the electron-two-ripplon coupling function which plays a crucial role in finding $\dot{Q}$. Different approaches to finding $W(q)$ are discussed in literature.

According to the previously given analysis [15], for electron scattering within the ground surface level we can represent $W(q)$ as a sum of long-wavelength and shortwavelength parts:

$$
W(q)=W_{(-)}(q) \theta\left(q_{0}-q\right)+W_{(+)}(q) \theta\left(q-q_{0}\right) .
$$

Here $\theta(x)$ is the unit step-function, $q_{0}=2 \sqrt{\kappa_{0} \gamma_{1}} \simeq$ $\simeq 1.6 \cdot 10^{7} \mathrm{~cm}^{-1}$, and

$$
\begin{gathered}
W_{(-)}(q)=W_{0}=4 \kappa_{0} \frac{\hbar^{2} \gamma_{1}^{2}}{2 m} ; \\
W_{(+)}(q)=\frac{\hbar^{2} \gamma_{1}^{2} q^{2}}{2 m}\left[1-\frac{\gamma_{0}}{\gamma_{1}} \chi\left(\frac{q}{2 \gamma_{1}}\right)\right] .
\end{gathered}
$$

The function $\chi(x)$ has a rather cumbersome form:

$$
\chi(\zeta)=\frac{4}{x^{2}} \int_{0}^{\infty}\left[1-\frac{\eta^{2} \zeta^{2}}{2} K_{2}(\eta x)\right] \frac{\exp (-\eta)}{\eta} d \eta,
$$

where $K_{n}(x)$ is the modified Bessel function of the second kind.

Placing Eq. (14) into Eq. (13), we arrive to the following expression for the energy transferred to ripplons per unit time:

$$
\dot{Q}=T_{e} \widetilde{v}_{\infty}\left(T_{e}\right) \Phi\left(\frac{T_{e}}{T}\right)
$$

with

$$
\begin{gathered}
\Phi(x)=\frac{1}{\Gamma\left(\frac{5}{3}\right)}\left\{\left(\frac{\gamma_{1}}{\kappa_{0}}\right)^{2}\left(\frac{q_{E}}{2 \gamma_{1}}\right)^{4} \times\right. \\
\times \int_{0}^{y_{0}} y^{\frac{10}{3}}\left[1-\frac{\gamma_{0}}{\gamma_{1}} \chi\left(\frac{q_{E}}{2 \gamma_{1}} y^{2 / 3}\right)\right]^{2} \frac{\left(\mathrm{e}^{-y}-\mathrm{e}^{-x y}\right)}{\left(1-\mathrm{e}^{-x y / 2}\right)^{2}} d y+ \\
\left.+\int_{y_{0}}^{\infty} y^{\frac{2}{3}} \frac{\left(\mathrm{e}^{-y}-\mathrm{e}^{-x y}\right)}{\left(1-\mathrm{e}^{-x y / 2}\right)^{2}} d y\right\} \\
y_{0}=\frac{2 \hbar \omega_{\mathbf{q}_{0}}}{T_{e}}, \quad q_{E}=\left(\frac{T_{e}}{2 \hbar}\right)^{\frac{2}{3}}\left(\frac{\rho}{\alpha}\right)^{\frac{1}{3}} \\
\widetilde{v}_{\infty}\left(T_{e}\right)=\frac{\Gamma\left(\frac{5}{3}\right)}{6 \pi \cdot 2^{2 / 3}} \cdot \frac{m W_{0}^{2} T_{e}^{2 / 3}}{\hbar^{5 / 3} \rho^{2 / 3} \alpha^{4 / 3}} .
\end{gathered}
$$

The function $\Phi\left(T_{e} / T\right)$ is plotted in Fig. 3 for $T=0.52 \mathrm{~K}$ (solid line). The function

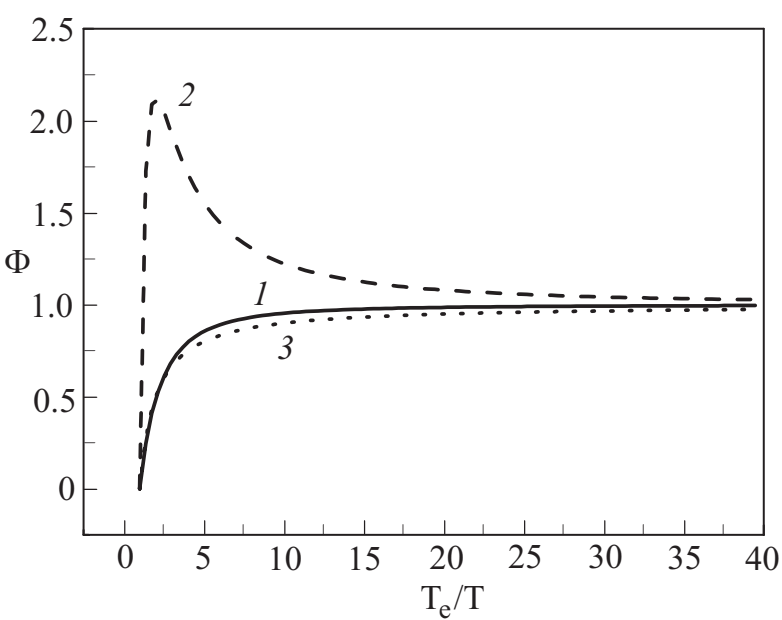

Fig. 3. The functions $\Phi\left(T_{e} / T\right)(1), F_{1}\left(T_{e} / T\right)(2)$, and $F_{2}\left(T_{e} / T\right)$ (3) for $T=0.52 \mathrm{~K}$.

$$
F_{1}(x)=\frac{1}{\Gamma\left(\frac{5}{3}\right)} \int_{0}^{\infty} y^{\frac{2}{3}} \frac{\left(\mathrm{e}^{-y}-\mathrm{e}^{-x y}\right)}{\left(1-\mathrm{e}^{-x y / 2}\right)^{2}} d y
$$

previously obtained in the Ref. 14 is also plotted in this figure as a dashed line. It is interesting to note that the solid line representing $\Phi\left(T_{e} / T\right)$ is numerically close to the intuitively introduced function $F_{2}\left(T_{e} / T\right)=1-T / T_{e}$ (dotted line) which was widely used in previous theoretical treatments of hot SEs. Still, at $x \sim 1$ the difference between functions $\Phi(x)$ and $F_{2}(x)$ is quite noticeable. In present work we use Eq. (15) with $\Phi(x)$ of Eq. (16).

Strictly speaking, for high enough $E_{\perp}$, the one-ripplon process contribution into energy relaxation frequency should be considered in addition to that of two-ripplon processes. The estimates [11] show however that one can disregard the one-ripplon contribution to energy relaxation for holding fields $E_{\perp}<1500 \mathrm{~V} / \mathrm{cm}$ of the range applied in the actual experiment. Only for $E_{\perp}$ close to $3000 \mathrm{~V} / \mathrm{cm}$ the one-ripplon processes do contribute in energy relaxation. For this reason we restrict ourselves to energy relaxation limited by two-ripplon processes.

\section{Results and discussion}

Typical experimental results for SE mobility as a function of driving electric field are plotted in Fig. 4 for $T=0.52 \mathrm{~K}$ and different holding fields. The electron density is $1.46 \cdot 10^{8} \mathrm{~cm}^{-2}$. Similar data were obtained for other temperatures and electron densities. The experimental data (points) are compared with theoretical curves calculated for single-electron approximation (solid line) and the above given many-electron treatment (dotted line) assuming that SEs populate only the ground surface level $(l=1)$. Figure 4 displays results obtained for different holding fields. Strong discrepancy between experimental data and theoretical curves observed at low holding fields 

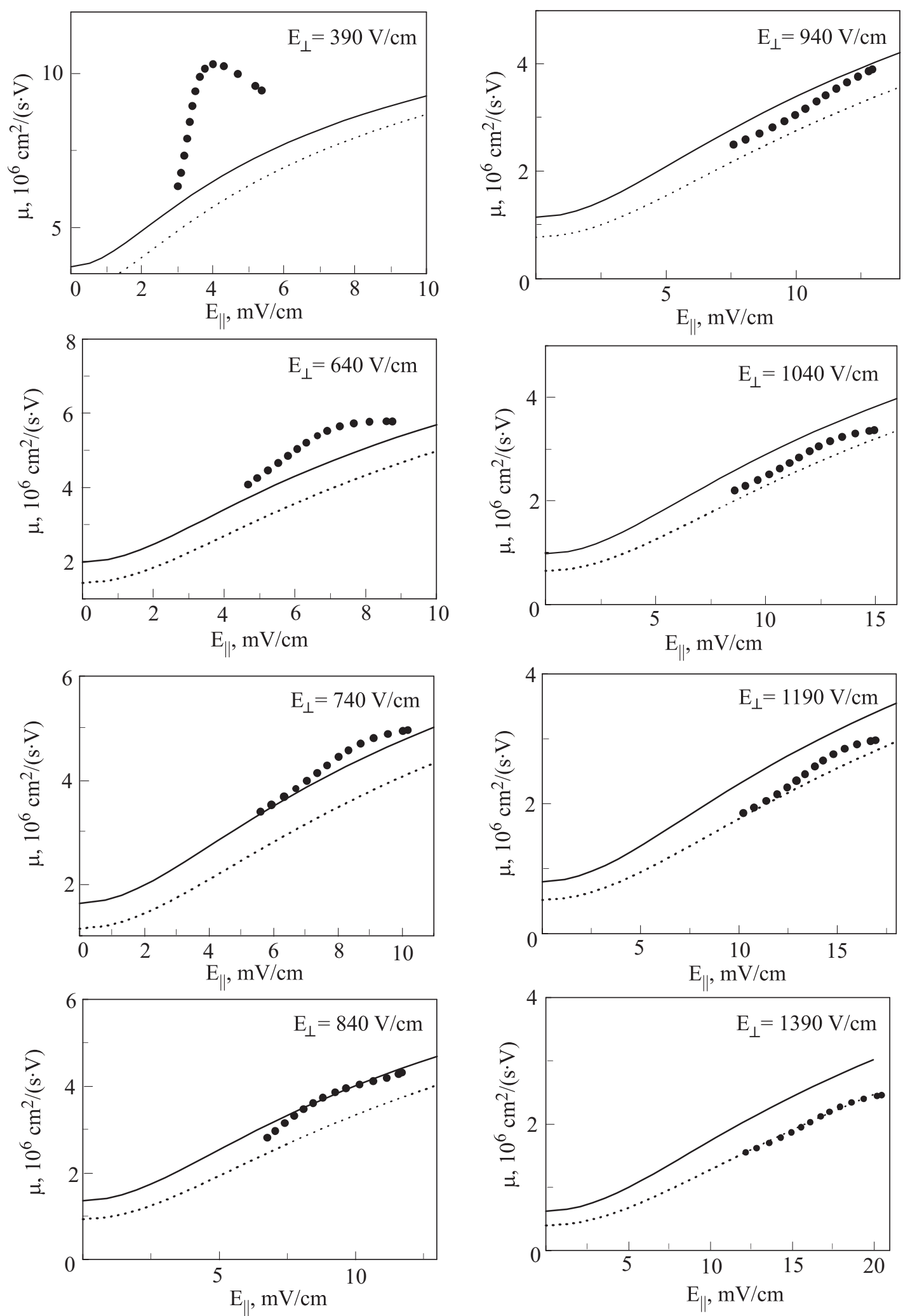

Fig. 4. The SE mobility as a function of driving electric field for different holding fields. Points are the experimental data. The solid and dotted lines correspond to theoretical calculations in one-electron and complete control approximations, respectively.

is attributed to effect of SE «evaporation» from the ground level to higher surface levels $(l>1)$. Sharp increase in $\mu$ with $E_{\|}$, a local maximum and the following slow decrease are in qualitative agreement with calculations performed previously for the all-level treatment under conditions $T=0.6 \mathrm{~K}$ and $E_{\perp}=300 \mathrm{~V} / \mathrm{cm}[10]$. Still, 

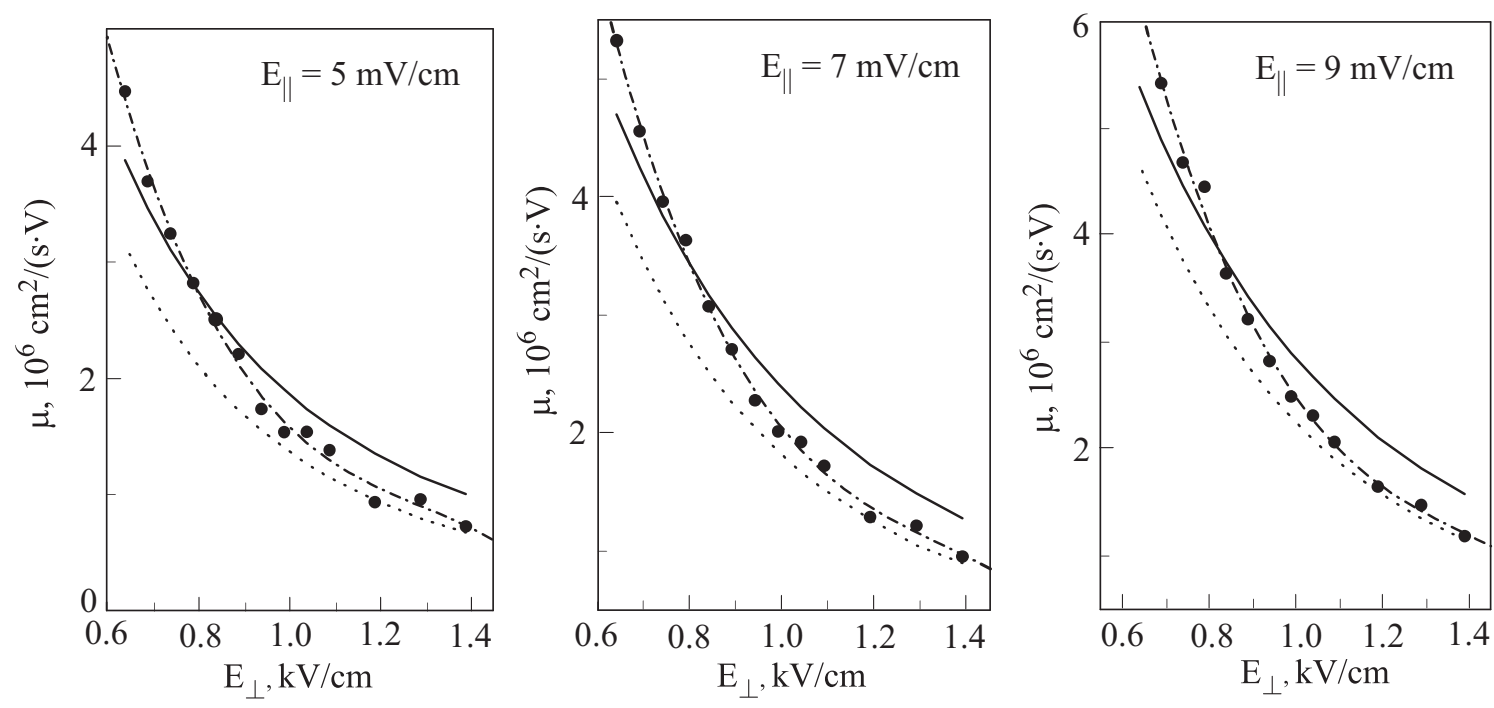

Fig. 5. The SE mobility as a function of holding electric field for three values of driving field. Points are the experimental data, dash-and-dotted lines are a result of the approximation. The solid and dotted lines correspond to theoretical calculations in one-electron and complete control approximations, respectively.

in those calculations there was also a substantial decrease in the ground level mobility of hot electrons caused by employment of a low-temperature approximation for electron-ripplon coupling $\varphi(y)$. Similar sharp increase in the nonlinear mobility and complicated nonohmic curves induced by SE evaporation were theoretically obtained in Ref. 9. The discrepancy noted above could also be attributed to the lack of experimental accuracy under high electron mobility conditions leading to very small values of the phase shift which are of the same order as the sensitivity of experimental procedure.

When increasing the holding field the experimental data become closer to theoretical curves obtained for the one level model. For $E_{\perp} \simeq 740-840 \mathrm{~V} / \mathrm{cm}$, data are close the theoretical curve calculated in the single-electron approach. The most interesting behavior of mobility is ob-

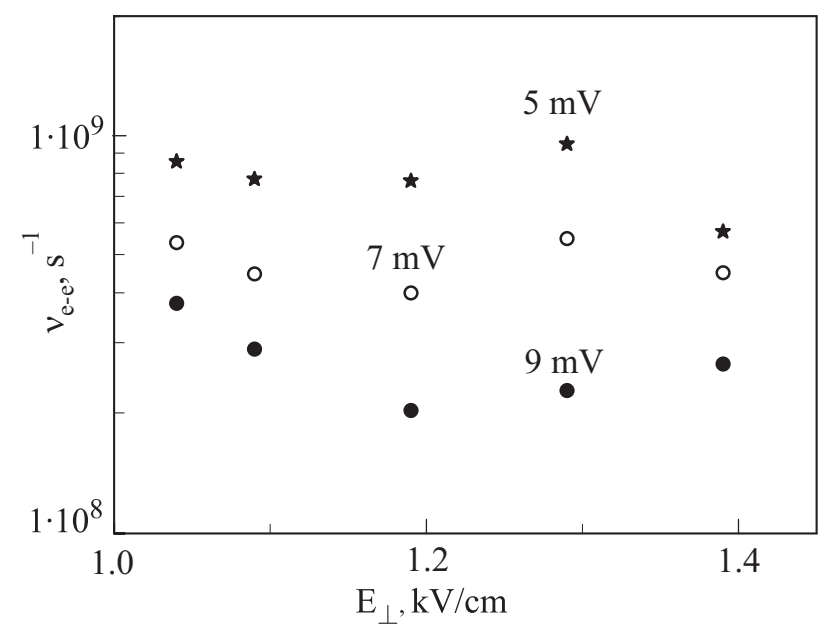

Fig. 6. The frequency of inter-electron collisions $v_{e-e}$ as a function of holding electric field for three values of driving field. served at higher $E_{\perp}$. The experimental data enter the interval between two theoretical curves and coincide with the complete control curve at highest holding fields. A similar tendency was observed in Ref. 7 where SE mobility was measured in the linear transport regime.

To make a situation more clear, in Fig. 5 we depicted the dependencies $\mu\left(E_{\perp}\right)$ measured for fixed values of $E_{\|}$. The graphics illustrate the transition between two scattering regimes under increasing the holding potential. One should emphasize the transition is observed for the range of holding field of $800-1300 \mathrm{~V} / \mathrm{cm}$ for all three driving fields.

As we mentioned above, the general expression for SE mobility was obtained in Ref. 12 for arbitrary relation between the frequency $v_{e-e}$ of inter-electron collisions and relaxation frequencies in one-electron and complete control regimes. Adjusting our experimental data to Eq. (11) we can estimate the frequency $v_{e-e}$. The results are presented in Fig. 6 for $1000 \mathrm{~V} / \mathrm{cm}<E_{\perp}<1400 \mathrm{~V} / \mathrm{cm}$.

Note that the accuracy of experiment is not satisfactory for holding field close or smaller than $1000 \mathrm{~V} / \mathrm{cm}$ where the experimental points are rather close to the theoretical curve of one-electron approach. For $E_{\perp}$ higher than $1000 \mathrm{~V} / \mathrm{cm}$, we observed the $v_{e-e}$ which depends weakly on holding field (decreases a little for holding fields close to $1000 \mathrm{~V} / \mathrm{cm}$ and then increases in the range of holding field where experimental accuracy is satisfactory) and decreases under increasing $E_{\|}$. The values of $v_{e-e}$ change in the range $\sim(2-8) \cdot 10^{8} \mathrm{~s}^{-1}$.

To elucidate the problem of energy balance in the SE system, we applied the approach where experimental data for $\mu\left(E_{\|}\right)$were compared with theoretical curves calculated using two approaches for $\mu\left(T_{e}\right)$ (open and solid circles represent the single-electron and complete control approaches, respectively). Such a comparison gives a 

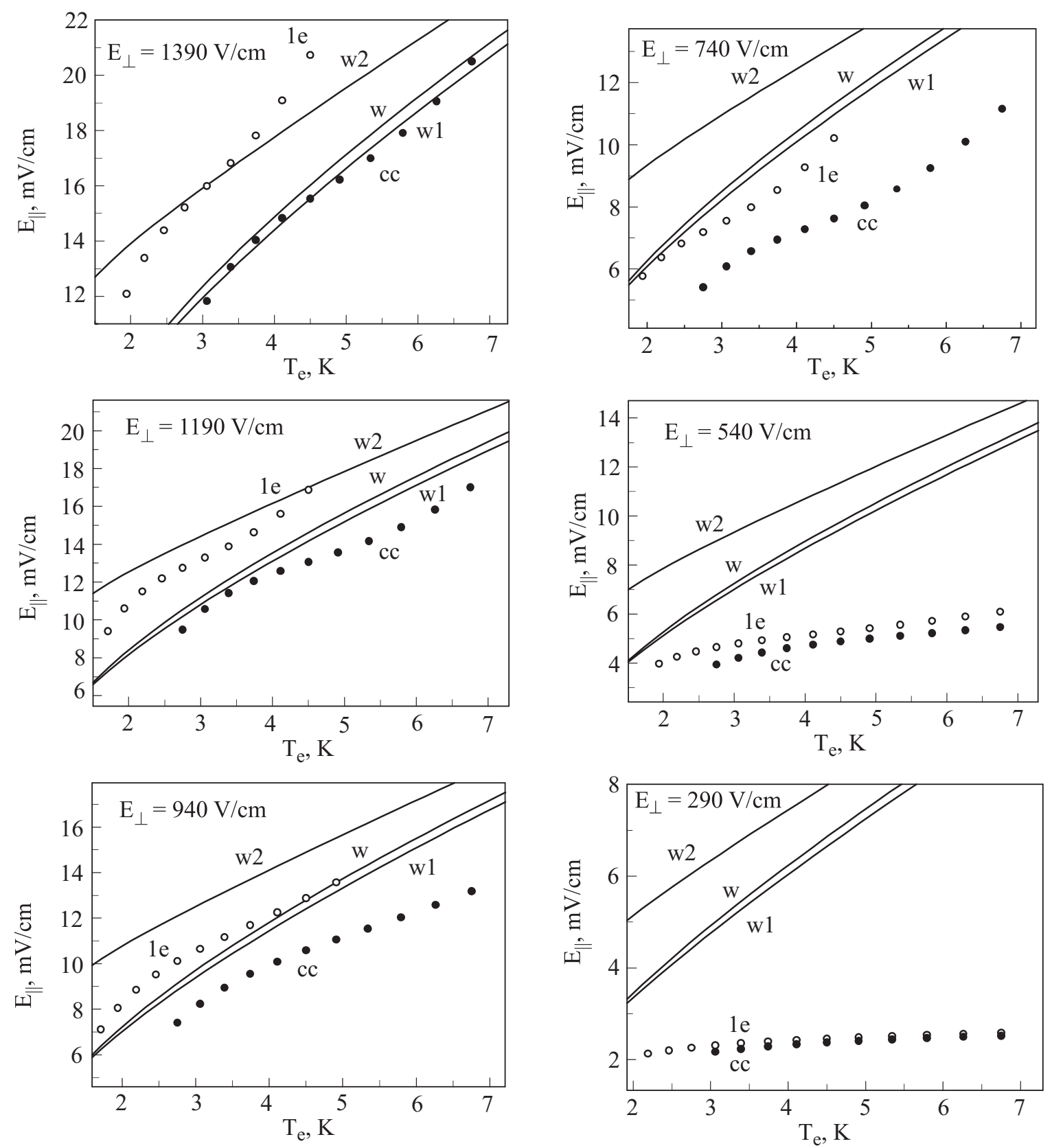

Fig. 7. The driving electric field as a function of effective electron temperature. Points are the result of calculation using experimental data and one-electron (open circle) and complete control (solid circle) approaches. The solid lines are the theoretical curves $T_{e}\left(E_{\|}\right)$.

possibility to obtain the dependence $E_{\|}\left(T_{e}\right)$ (Fig. 7). The same figure contains the theoretical curves $T_{e}\left(E_{\|}\right)$of two mobility regimes obtained from the energy balance equation (12) using different approximations for $\Phi\left(T_{e} / T\right)$ presented in Fig. 3. One can see that the one-level theory and experiment disagree strongly at low holding fields. The same behavior is observed in Fig. 4. At higher holding fields the agreement between experimental points and theoretical curves becomes better. Note that the best agreement is attained for the function $\Phi\left(T_{e} / T\right)$ of Eq. (16).

In Fig. 8, we plotted experimental dependencies $E_{\|}\left(T_{e}\right)$ obtained under assumption that $T_{e}$ is calculated using either $\mu^{(1 e)}\left(T_{e}\right)$ or $\mu^{(m e)}\left(T_{e}\right)$. At low holding fields where the experimental accuracy is not high we observe the practical independence of driving field in the whole range of effective electron temperature. In other words the effective electron temperature increases sharply with the driving electric field. It means that the possibility to overheat the electron sheet is very high. When increasing the holding potential we enter the range of holding fields where SEs mostly occupy the ground surface level and the one-level theory gives the adequate description of SE mobility. Under such a condition the dependencies $E_{\|}\left(T_{e}\right)$ and consequently $T_{e}\left(E_{\|}\right)$are increasing functions in agreement with theoretical estimation of Ref. 11 . 

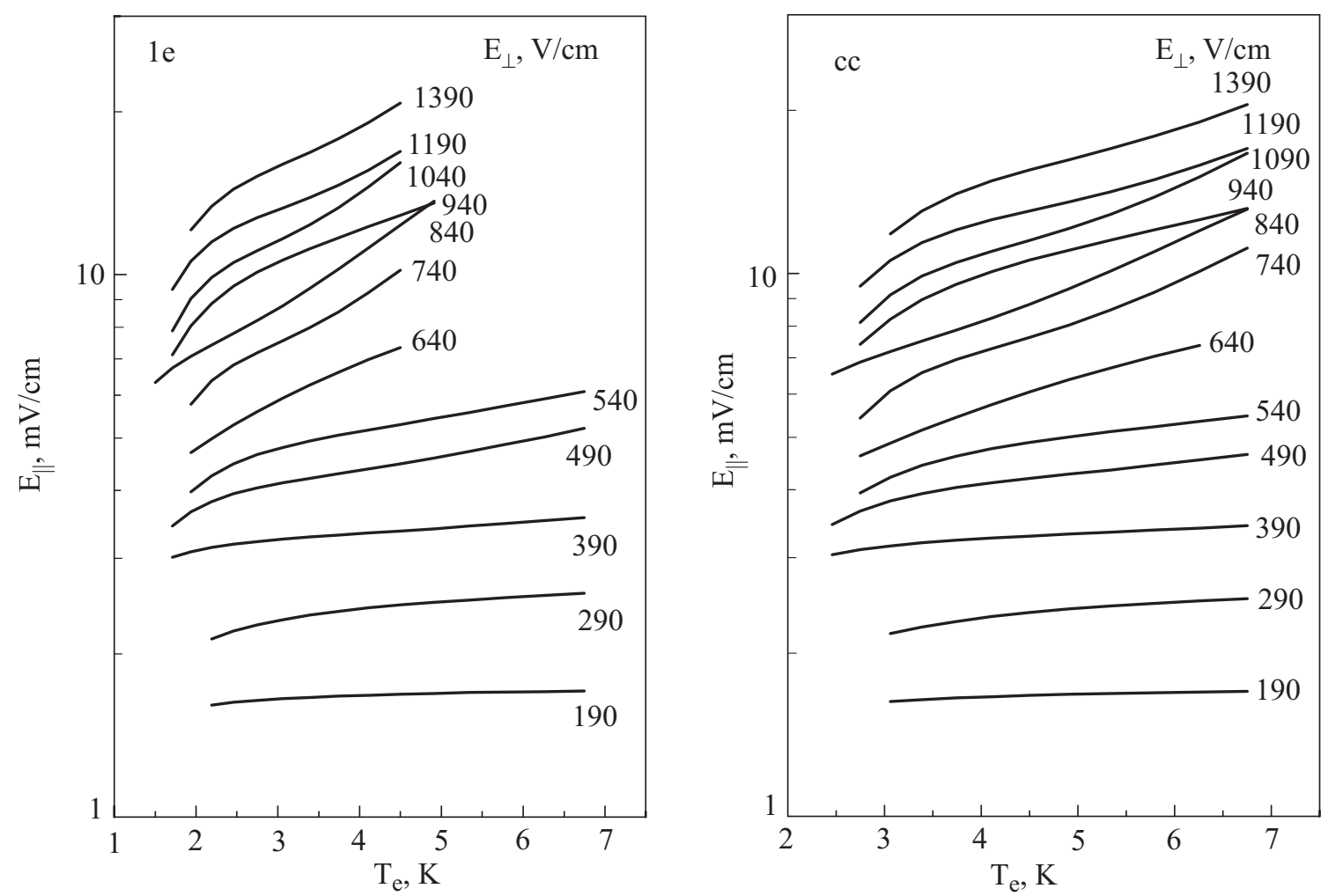

Fig. 8. The same as in Fig. 7 for energy balance equation solved either for $\mu^{(1 e)}\left(T_{e}\right)$ or $\mu^{(m e)}\left(T_{e}\right)$.

\section{Conclusion}

We presented the results of experimental and theoretical studies of nonlinear mobility of SEs along the liquid-vapor interface limited by electron-ripplon scattering under conditions where the effective electron temperature is substantially higher than the ambient temperature. It is shown that for high holding electric fields, mobility of SEs occupying the ground surface level increases with the driving electric field in agreement with the theoretical model of highly correlated electron gas. For low holding fields, electron evaporation to higher surface levels induced by heating becomes important which complicates experimental curves and theoretical analysis. Energy relaxation of SE system is shown to be in agreement with the model of SE scattering by pairs of short-wavelength ripplons $q, q^{\prime}>>2 k$, which takes into account the reduction in electron-two-ripplon coupling induced by transition to the adiabatic scattering regime [Eq. (16)]. The experimental technique and theoretical formalism developed can be used for further studies of nonlinear transport of SEs on liquid helium.

The work is partially supported by STCU through the Project 3718.
1. Yu.P. Monarkha and K. Kono, Two-Dimensional Coulomb Liquids and Solids, Springer, Berlin (2004).

2. V.E. Sivokon, V.V. Dotsenko, S.S. Sokolov, Yu.Z. Kovdrya, and V.N. Grigor'ev, Fiz. Nizk. Temp. 22, 715 (1996) [Low Temp. Phys. 22, 549 (1996)].

3. V. Syvokon, Y. Monarkha, K. Nasyedkin, and S. Sokolov, J. Low Temp. Phys. 148, 169 (2007).

4. C.L. Zipfel, T.R. Brown, and C.C. Grimes, Phys. Rev. Lett. 37, 1760 (1976).

5. V.B. Shikin and Yu.P. Monarkha, J. Low Temp. Phys. 16, 193 (1974).

6. Yu.P. Monarkha and S.S. Sokolov, Fiz. Nizk. Temp. 4, 685 (1978) [Sov. J. Low Temp. Phys. 4, 327 (1978)].

7. V.A. Buntar', Yu.Z. Kovdrya, V.N. Grigor'ev, Yu.P. Monarkha, and S.S. Sokolov, Fiz. Nizk. Temp. 13, 789 (1987) [Sov. J. Low Temp. Phys. 13, 451 (1987)].

8. V.A. Buntar and S.S. Sokolov, Fiz. Nizk. Temp. 16, 845 (1990) [Sov. J. Low Temp. Phys. 16, 497 (1990)].

9. Yu.P. Monarkha and S.S. Sokolov, Fiz. Nizk. Temp. 5, 1283 (1979) [Sov. J. Low Temp. Phys. 5, 605 (1979)].

10. M. Saitoh and T. Aoki, J. Phys. Soc. Jpn. 44, 71 (1978).

11. Yu.P. Monarkha, Fiz. Nizk. Temp. 5, 994 (1979) [Sov. J. Low Temp. Phys. 5, 470 (1979)].

12. I.N. Adamenko, A.V. Zhukov, and K.E. Nemchenko, Fiz. Nizk. Temp. 26, 631 (2000) [Low Temp. Phys. 26, 459 (2000)].

13. Yu.P. Monarkha, Fiz. Nizk. Temp. 4, 1093 (1978) [Sov. J. Low Temp. Phys. 4, 515 (1978)].

14. Yu.M. Vil'k and Yu.P. Monarkha, Fiz. Nizk. Temp. 15, 235 (1989) [Sov. J. Low Temp. Phys. 15, 131 (1989)].

15. Yu.P. Monarkha, Fiz. Nizk. Temp. 19, 235 (1993) [Low Temp. Phys. 19, 162 (1993)]. 